\title{
A Topical Application Containing Sucralfate, Zinc Oxide and Ketoconazole Provides High Patient Satisfaction in the Treatment of Intertrigo
}

\author{
Craig G. Burkhart ${ }^{*}, 1$, Curtis Black ${ }^{2}$ and Craig N. Burkhart ${ }^{3}$ \\ ${ }^{1}$ University of Toledo College of Medicine, USA \\ ${ }^{2}$ University of Toledo College of Pharmacy, Toledo, Ohio, USA \\ ${ }^{3}$ Department of Dermatology, University of North Carolina at Chapel Hill, Chapel Hill, North Carolina, USA
}

\begin{abstract}
Intertrigo is a superficial inflammatory dermatitis involving juxtaposed skin surfaces subjected to friction, heat, moisture, and maceration. In response to encouraging reports of topical sucralfate's effect on epithelialization of wounds as well as its bacteriostatic property, a clinical trial evaluating its possible role as a topical agent for intertrigo was initiated. For expected beneficial effects, zinc oxide and ketoconazole were added with sucralfate to the vehicle. The study was an open-label comparison designed to examine patient satisfaction with topical sucralfate $v s$ the patients' prior therapies for their condition. At the conclusion of our pilot study, patients completed a survey questionnaire so that we could assess the value of this product in clinical usage. By all parameters queried, patient satisfaction was very high with this product for their condition. It is conjectured that such a formulation might be useful also for diaper rashes, diaper wipes, blisters, and open skin sores.
\end{abstract}

Sucralfate is a synthetic, water-insoluble, sulfated disaccharide conjugated with aluminum salt. It was developed in the early 1980 s as an orally effective mucoprotective agent for the treatment of gastric and duodenal ulcers. The negatively charged polyanions of sucralfate form polyvalent bonds with the positively charged mucoproteins of the damaged epidermis or mucosal surfaces. Thus, the drug adheres to the epithelial proteins at the ulcer site. This then forms a protective coating against the environment. Sucralfate increases both epidermal growth factor and basic fibroblast growth factor concentration in wounds.

After the initial work with topical sucralfate in the management of resistant peristomal and perineal excoriations, various workers have tried this drug on stomatitis, canker sores, healing of decubitus ulcers, second and third degree burns, keratoconjunctivitis, diaper dermatitis, perianal skin irritation, moist desquamation during radiotherapy, oral management in patients with epidermolysis bullosa, and radiation proctitis [1-4].

Orally the only common side effect is the occasional constipation and a report of aluminum toxicity. Because of this latter concern, serum aluminum level of significantly burned patients using a $7 \%$ sucralfate cream to their skin twice daily has been specifically studied. This scientific study did not show any detectable amount of aluminum in their blood samples [1]. In that same study, of the 85 burn patients treated with sucralfate, none developed any burn wound sepsis and the agent was found to contribute in controlling the bacterial population.

*Address correspondence to this author at the University of Toledo School of Medicine, 5600 Monroe Street, Suite 106B, Sylvania, OH 43560, USA; Tel: 419-885-3403; Fax: 419-885-3401; E-mail: cgbakb@aol.com
Encouraging reports of topical sucralfate's effect on epithelialization of wounds along with its bacteriostatic property have led us to carry out a trial to evaluate its role as a topical agent in the treatment of intertrigo. In this study we added zinc oxide and ketoconazole to the vehicle. At the conclusion of our pilot study we asked the patients to complete a survey questionnaire so that we could assess the value of this product in clinical usage. It is conjectured that such a formulation might be useful also for diaper rashes, diaper wipes, blisters, and open skin sores.

\section{STUDY DESIGN}

This study was an open-label comparison designed to examine patient satisfaction with topical sucralfate $v s$ the patients' prior therapies for their condition. Eight adult patients, ranging in age from 36 to 64 and demonstrating intertrigo with symmetrical, erythematous eruption of the apposed surfaces of the inframammary and/or crural and abdominal skin folds, were recruited from September 2006 to July 2008 to participate in the study.

All participants were given sufficient quantities of the sucralfate topical agent to apply twice daily to the affected skin regions. The formulated agent contained $20 \%$ zinc oxide, $7 \%$ sucralfate, and $1 \%$ ketoconazole in an adsorption base. After 28 days of treatment, patients returned to the office and completed a questionnaire survey.

Study subjects were excluded if they have extensive dermatitis, or if they had, within the prior month, used any oral or injectable steroids.

Patients were seen at baseline and at four weeks. The survey questions posed to the patients are presented in Table 1. Utilizing a 10-point scale ('definitely didn't help' to 'did a fantastic job'), patients were asked to rate their responses to 
Table 1. Survey Questions Posed to Patients Using Sucralfate Topically to Affected Skin Areas After 4 Weeks of Therapy

1. Did you find the use of sucralfate helpful in reducing the size of the affected skin condition over previous therapy? (grade on a scale of 1-10, with 1 meaning that the product did not help at all, and 10 meaning that the product was fantastic)
1
2
3
4
5
6
7
8
9
10

2. Did you find the use of sucralfate helpful in reducing symptoms associated with the skin condition over previous therapy? (grade on a scale of 1-10, with 1 meaning that the product did not help at all, and 10 meaning that the product was fantastic)
1
2
3
4
56
7
8
9
10

3. Did you find the use of sucralfate helpful in reducing any signs of infection (pus, redness, warmth) associated with the skin condition over previous therapy? (grade on a scale of 1-10, with 1 meaning that the product did not help at all, and 10 meaning that the product was fantastic)
1
2
3
$4 \quad 5$
6
$8 \quad 9$
10

4. Did you find the use of sucralfate irritating to the skin where the skin condition is present? (grade on a scale of 1-10, with 1 meaning that the product caused a lot of irritation, and 10 meaning that the product did not cause any irritation)
2
3
4
$5 \quad 6 \quad 7$
8
$9 \quad 10$

5. Did you find the use of sucralfate helpful in speeding your recovery over previous therapy? (grade on a scale of 1-10, with 1 meaning that the product did not help at all, and 10 meaning that the product was fantastic)

$\begin{array}{llllllllll}1 & 2 & 3 & 4 & 5 & 6 & 7 & 8 & 9 & 10\end{array}$

6. Please list your previous topical therapy prior to this study

several parameters. The study was approved by the Promedica Institutional Review Board, Toledo, Ohio.

\section{RESULTS}

Questionnaires were returned by all eight patients, seven of whom were female. The patients' ratings for the first five questions were tabulated and the averages are shown in Table 2. All patients stated improvement of their rash with the preparation, and all asked for additional quantities of the product on their return visit. The top responses were in terms of speed of clinical recovery and reduction of size of affected skin. Previous treatment listed in response to the last question included topical antifungal, topical anti-yeast preparations, topical steroids, topical immunomodulators, and a host of home remedies. Of note, the two patients who recently used topical steroids noted mild irritation from the sucralfate preparation.

\section{CONCLUSIONS}

Intertrigo is a superficial inflammatory dermatitis involving juxtaposed skin surfaces subjected to friction, heat, moisture, and maceration. Although intertrigo can be found in men and women of all ages, it is most commonly seen in obese middle-aged women or those with large breasts. Hot, humid environments typically exacerbate the condition. Other contributing factors include diabetes mellitus, heavy perspiration, vaginal discharge, urinary incontinence, and the use of topical steroids in flexural areas. Increased moisture leads to the overgrowth of Candida albicans in the skin folds, contributing to the severity of the eruption.
Clinically, intertrigo presents with a symmetrical, erythematous eruption of the apposed surfaces of the inframammary and/or crural and abdominal skin folds. The eruption is well-marginated, often with peripheral satellite vesicles or pustules. The severity of the rash can vary, and edema, fissuring, erosion, weeping, and a resultant offensive odor can occur. Burning, itching and pain at the affected sites is not unusual.

The bacterial and yeast organisms that proliferate in these body regions are typically overgrowth of normal, transient flora. The overgrowth of bacteria in intertrigo is usually corrected merely by maintaining a dry environment in the affected areas. This can be accomplished with air-drying, and powder. For patients with large breasts, a good support bra is recommended, and frequent changing is indicated if the fabric becomes moist.

Sucralfate proved most beneficial for our patients with intertrigo. The formulation for our study was $20 \%$ zinc oxide, $7 \%$ sucralfate, and $1 \%$ ketoconazole in an adsorption base. The vehicle included cholesterol, white wax, and mineral oil. This formulation proved non-irritating to open sores which allowed application of the sucralfate to abraded skin with very good tolerance and therapeutic benefit. It did not require any bandage to hold in place.

Limitations to the study included the low number of patients entered into the study. Additionally, the participants were not taken randomly, but were individuals who were not responding to their present therapy.

Table 2. Averages for Patient Assessment of Clinical Parameters on the 10-Point Scale Used in the Survey

\begin{tabular}{|l|l|}
\hline \multicolumn{1}{|c|}{ Query } & Average Score \\
\hline \hline How well was sucralfate in reducing the size of the affected skin condition over previous therapy? & 9.0 \\
\hline How well was sucralfate helpful in reducing symptoms over previous therapy? & 8.4 \\
\hline How well was sucralfate in reducing signs of infection over previous therapy? & 8.8 \\
\hline Was sucralfate irritating to the skin? & 7.6 \\
\hline Was sucralfate helpful in speeding your recovery over previous therapy? & 9.0 \\
\hline
\end{tabular}


In the future, this preparation might prove useful to assist in the healing of many types of wounds and ulcers, skin fissures, and as a diaper wipe. A slight variance in the formulation would allow the addition of an antibiotic for crusted, infected lesions. For fissures and/or cracked skin, one might use an ointment preparation which contains proteins to assist the reformation of the epidermal tissues. The mineral oil content can be adjusted to allow for variance in base consistency.

In short, sucralfate proved to have very high patient satisfaction in the treatment of intertrigo. Similar preparations may have benefit for a range of dermatological entities.

\section{REFERENCES}

[1] Banati A, Chowdhury SR, Mazumder S. Topical use of sucralfate cream in second and third degree burns. Burns 2001; 27: 465-9.

[2] Etiz D, Erkal HS, Serin M, et al. Clinical and histopathological evaluation of sucralfate in prevention of oral mucositis induced by radiation therapy in patients with head and neck malignancies. Oral Oncol 2000; 36: 116-20.

[3] Lyon CC, Stapleton M, Smith AJ, Griffiths CEM, Beck MH. Topical sucralfate in the management of peristomal skin disease: an open study. Clin Exp Dermatol 2000; 25: 584-8.

[4] Burkhart CG. Add sucralfate to list of aphthous ulcer treatments. Cutis 2002; 270: 5 . 